\title{
Tiefe Hirnstimulation bessert die Tics
}

Fragestellung: Ist eine tiefe Hirnstimulation im anteromedialen Globus pallidus internus effektiv beim Gilles-de-la-Tourette Syndrom?

Hintergrund: Bisher wurde in der Literatur von über 60 Patienten mit Tourette-Syndrom und tiefer Hirnstimulation berichtet. Etwa $90 \%$ profitierten von der Behandlung mit einer Reduktion (quantitativ und/oder qualitativ) der Tics. Dabei wurden neun verschiedene Hirnregionen stimuliert, und es ist bis heute offen, bei welcher Region die Erfolgsaussichten am größten sind. Aussichtsreichste Kandidaten sind der Thalamus und vor allem der Globus pallidus internus als wichtigster Ausgang der Basalganglien zum Thalamus.

Patienten und Methodik: Bei elf Patienten mit „therapierefraktärem“ Gilles-de-la-Tourette Syndrom (drei bis sechs erfolglose medikamentöse

Cannon E, Silburn P, Coyne T et al. Deep brain stimulation of anteromedial globus pallidus interna for severe Tourette's syndrome. Am J Psychiatry 2012; 169: $860-6$ Therapieversuche) wurde bilateral im anteromedialen Globus pallidus ein Stimulationssystem (Medtronic) implantiert. Die Stimulationsparameter wurden individuell in Abhängigkeit von Wir- kung und Nebenwirkung adjustiert. Die Yale Global Tic Severity Scale wurde als Maß für den Schweregrad des Tourette-Syndroms vor der Implantation sowie einen und drei Monate danach erfasst, außerdem am Ende der Beobachtungszeit (im Mittel 14 Monate). Zwang, Depressivität und Funktionsniveau wurden als sekundäre Erfolgskriterien überprüft. Prae-postVergleiche wurden statistisch mit dem Friedman- und dem Wilcoxon-Test evaluiert.

Ergebnisse: Sowohl motorische (48\% Reduktion bis zum letzten Untersuchungstermin) als auch vokale/phonetische Tics (56\% Reduktion) wurden statistisch signifikant reduziert. Zehn von den elf Patienten waren Responder. Die wesentliche Besserung trat rasch innerhalb des ersten Monates ein. Zwei Patienten wiesen vorübergehend vermehrt Angstsymptome auf, ein $\mathrm{Pa}$ tient brach die Therapie ab und bei drei Patienten traten technische Probleme auf. Als Nebeneffekt besserten sich auch alle komorbiden psychischen Störungen beziehungsweise Symptome signifikant.

Schlussfolgerungen: Die Autoren der Studie folgern aus ihren Untersuchungsergebnissen, dass die tiefe Hirnstimulation im anteromedialen Globus pallidus eine effektive und sichere Therapie des Tourette-Syndroms darstellt.

\section{- Kommentar von Prof. Dieter Ebert}

\section{Bei schwerem Tourette-Syndrom eine Erwägung wert}

Diese offene Studie liefert weitere Belege für die Wirksamkeit und Sicherheit der tiefen Hirnstimulation beim Tourette-Syndrom. Sie reicht aber noch nicht für eine klinisch verwertbare Therapieempfehlung. Es wurden nur wenige Patienten ohne Kontrollgruppen und ohne Verblindung untersucht. Therapieresistent wurde nicht operationalisiert, ein direkter Vergleich mit medikamentösen Verfahren steht noch aus und so auch eine Analyse, welche Patienten von welchen Verfahren profitieren. Auch die Frage nach dem Stimulationsort bleibt offen, auch wenn mit dieser Studie der anteromediale Globus pallidus vorläufig die Region ist mit den wahrscheinlich besten Er- gebnissen, ohne dass aber direkte Regionenvergleiche durchgeführt wurden.

Negative psychische Veränderungen einschließlich Suizidgedanken als gefürchtete Nebenwirkungen einer tiefen Hirnstimulation traten nicht auf, wobei die geringe Anzahl von elf Patienten keine Aussage zur Sicherheit zulässt.

Mit all diesen Einschränkungen lässt die Studie aber zumindest den Schluss zu, dass beim therapieresistenten, schweren und funktionell beeinträchtigenden Tourette-Syndrom die tiefe Hirnstimulation im anteromedialen Globus pallidus erwogen und mit dem Patienten diskutiert werden muss.
Weitere Infos auf

springermedizin.de

Informationen zum Thema Tics bei Kindern und Jugend-

lichen finden auf springermedizin.de in unserer Serie

"Arzneimittel in der psychiatrischen Therapie" unter

www.springermedizin.de/3200894
Prof. Dr. med. Dieter Ebert,

Freiburg i. Br.

Abteilung Psychiatrie und Psychotherapie,

Universitätsklinikum Freiburg i. Br.

E-Mail: dieter.ebert@uniklinik-freiburg.de 